\title{
Kyste hydatique du rein : Étude monocentrique de 15 cas
}

\author{
Youness El Harrech, MD ; Najib Abakka, MD ; Jihad El Anzaoui, MD ; Omar Ghoundale, MD ; Driss Touiti, MD
}

Service d'urologie, Hôpital militaire Avicenne, Marrakech, Maroc

Cite as: Can Urol Assoc J 2014;8(7-8):e531-5. http://dx.doi.org/10.5489/cuaj.1374 Published online August 11, 2014.

\section{Abstrait}

Objectif : Étudier le profil épidémiologique ainsi que les caractéristiques cliniques, radiologiques, et thérapeutiques du kyste hydatique du rein (KHR).

Méthodologie : Quinze cas de KHR colligés de 2004 à 2010 ont été revus. Les données cliniques, radiologiques (arbre urinaire sans préparation, échographie, tomodensitométrie [TDM]) et biologiques (éosinophilie et sérologie hydatique) ont été analysées, et le traitement instauré. L'évolution et les complications ont fait l'objet d'un suivi attentif.

Résultats : L'âge moyen de découverte du KHR est de 56,13 ans, avec une prédominance masculine (12 hommes, 3 femmes). La symptomatologie clinique est dominée par les lombalgies (60\% des cas). Une analyse de la sérologie hydatique a été demandée pour six patients et s'est révélée positive pour trois d'entre eux. L'abstention thérapeutique a été l'attitude adoptée par deux malades ayant un kyste hydatique de type 5 . Aucun patient n'a reçu de traitement médical seul. Treize patients $(86,66 \%)$ ont subi une intervention chirurgicale, dont une résection du dôme saillant pour 11 patients, une néphrectomie partielle pour 1 patient et une néphrectomie totale pour 1 autre patient dont le rein était totalement détruit. Aucune complication peropératoire ou postopératoire n'a été signalée, qu'il s'agisse de fistule urinaire ou d'infection de la cavité résiduelle. Aucune récidive n'a été constatée sur une période moyenne de deux ans.

Conclusion : Le KHR est une pathologie rare sans sémiologie spécifique. Si la chirurgie occupe une place primordiale dans le traitement de ces kystes, de nouvelles perspectives mini-invasives restent à explorer, tout particulièrement les traitements percutané et laparoscopique.

\section{Introduction}

Le kyste hydatique, ou hydatidose, est une anthropozoonose attribuable au développement de la forme larvaire du tænia Echinococcus granulosus chez l'homme. Il sévit à l'état endémique dans les pays du pourtour méditerranéen, dont le Maroc, et constitue un véritable problème de santé publique'. Tous les organes peuvent être atteints, mais plus de $90 \%$ des kystes hydatiques chez I'humain se localisent dans le foie et le poumon, alors que la localisation rénale ne représente que de $2 \%$ à $4 \%$ des $\operatorname{cas}^{2}$. Dans le présent article, nous proposons d'étudier le profil épidémiologique ainsi que les caractéristiques cliniques, radiologiques et thérapeutiques du KHR d'après les conclusions d'un essai rétrospectif réalisé dans un service d'urologie de la région de Marrakech.

\section{Méthodologie}

L'étude rétrospective qui nous occupe a porté sur l'ensemble des patients pris en charge pour un KHR de janvier 2004 à décembre 2010. Ont été retenues uniquement les données cliniques, radiologiques et anatomopathologiques de patients porteurs d'un KHR confirmé quels que soient leur âge et leur sexe. Ont été exclus les autres types de kystes rénaux.

Les données cliniques, radiologiques (arbre urinaire sans préparation, échographie, TDM) et biologiques (éosinophilie et sérologie hydatique) ont été analysées, et le traitement instauré. L'évolution et les complications ont fait l'objet d'un suivi attentif.

\section{Résultats}

Quinze patients ( 12 hommes et 3 femmes) âgés en moyenne de 56,13 ans (intervalle de 33 à 72 ans) ont été retenus. Dix patients (66,66 \%) étaient âgés de plus de 50 ans. Tous étaient d'origine rurale et avaient été en contact avec des chiens pendant leur enfance. La symptomatologie clinique était dominée par le syndrome douloureux $(60 \%)$, sous la forme de lombalgies chroniques intermittentes sans irradiation particulière. Aucun cas de colique néphrétique n'a été 
relevé. Aucun malade n'a signalé d'hématurie. Un seul patient a fait mention d'élimination de membranes blanchâtres, sans que cela puisse être confirmé à l'analyse des urines. Le KHR était asymptomatique chez deux patients et a été découvert fortuitement au cours d'une échographie abdominale.

L'examen physique a révélé une masse rénitente, sensible et mobile donnant un contact lombaire chez deux patients.

L'arbre urinaire sans préparation pratiqué auprès de huit patients a révélé des calcifications de l'aire rénale dans deux cas. L'échographie abdominale a été réalisée chez tous les patients et a objectivé le kyste dans tous les cas (fig. 1, fig. 2). Ces kystes ont été répertoriés selon la classification de Gharbi (tableau 1).

La TDM a été réalisée chez 9 patients (60\%) quand un doute diagnostique subsistait à l'échographie. Aucun patient n'a subi d'examen par IRM.

L'hyperéosinophilie sanguine n'a été constatée que chez trois patients. Une analyse de la sérologie hydatique par hémagglutination indirecte a été demandée pour six patients et s'est révélée positive pour trois d'entre eux. Aucun de nos patients ne présentait d'autre localisation hydatique associée.

L'abstention thérapeutique a été l'attitude adoptée par deux malades ayant un kyste hydatique de type 5. Aucun patient n'a reçu de traitement médical seul. Treize patients $(86,66 \%)$ ont subi une intervention chirurgicale par voie extrapéritonéale, dont une résection du dôme saillant pour 11 patients, une néphrectomie partielle pour 1 patient et une néphrectomie totale pour 1 autre patient dont le rein était totalement détruit.
Aucune complication peropératoire ou postopératoire n'a été signalée, qu'il s'agisse de fistule urinaire ou d'infection de la cavité résiduelle. La durée du séjour hospitalier a varié de trois à six jours, s'établissant en moyenne à quatre jours.

Trois patients ont reçu un traitement médical à base d'Albendazole pendant les trois mois qui ont suivi la chirurgie.

Les patients ont été revus en consultation avec évaluation clinique et échographique. Aucune récidive n'a été constatée sur une période moyenne de deux ans. L'ensemble des résultats sont résumés dans le tableau 2 .

\section{Discussion}

L'hydatidose est une parasitose cosmopolite, mais qui s'observe essentiellement dans les pays où subsiste l'élevage traditionnel des moutons. Elle est particulièrement répandue en Australie, en Nouvelle-Zélande, en Afrique du Nord et dans le pourtour du bassin méditerranéen ${ }^{3,4}$.

Le KHR représente de $2 \%$ à $3 \%$ des localisations viscérales et se classe en $3^{\mathrm{e}}$ position, après la localisation hépatique et pulmonaire ${ }^{2-5}$. L'homme représente une impasse biologique au cours du cycle évolutif de l'Echinococcus granulosus. Il peut se contaminer lui-même en ingérant des aliments souillés par des œufs de cestode provenant de déjections de chien ou directement lors d'un contact avec un chien ${ }^{6}$. Tous les patients de notre série étaient d'origine rurale et avaient déjà été en contact avec des chiens pendant leur enfance.

Selon certaines études, cette pathologie est l'apanage des jeunes adultes dont l'âge moyen varie de 36 à 49,5 ans $^{4-6}$. Dans

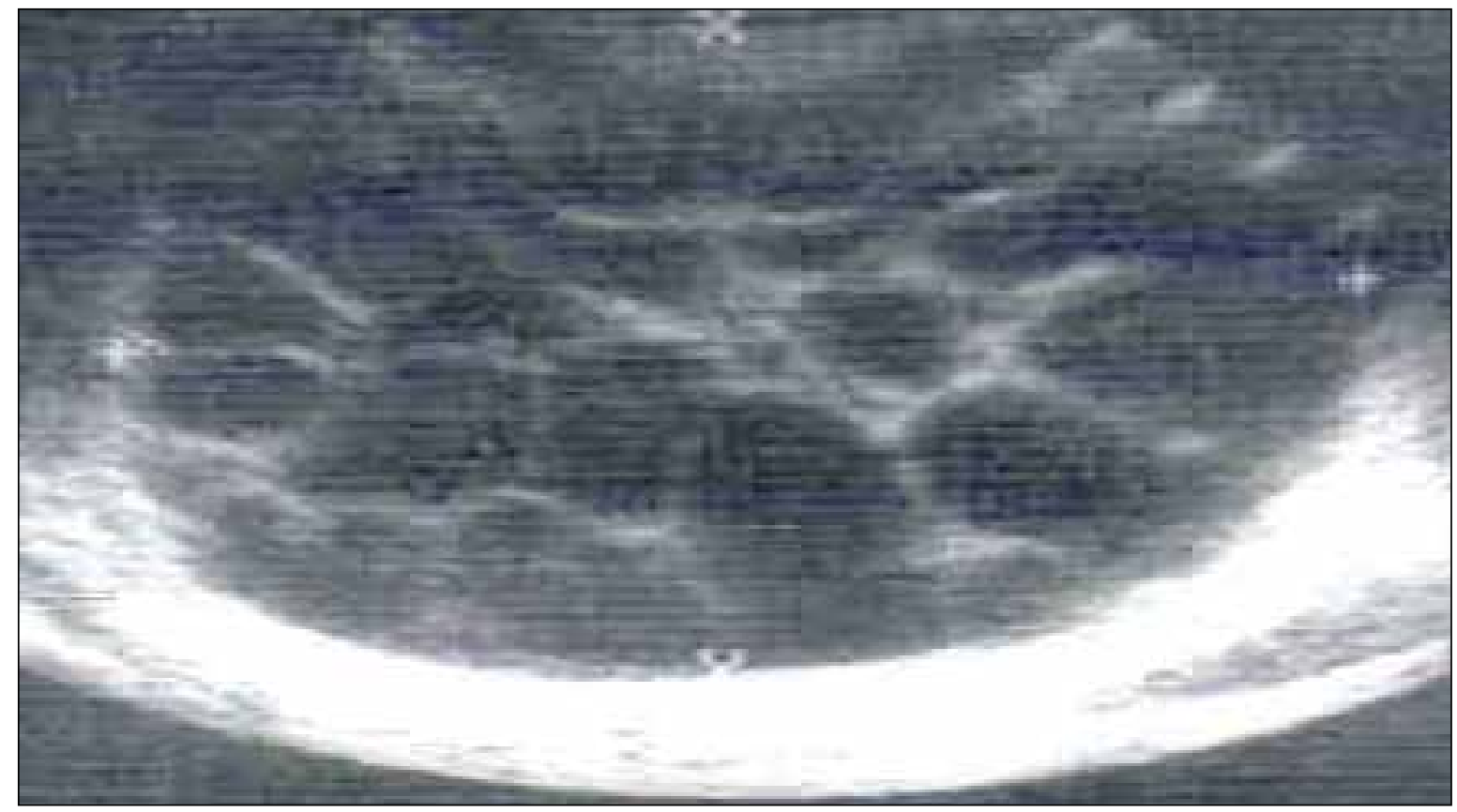

Fig. 1. Aspect échographique d'un kyste hydatique multivésiculaire de type 3. 


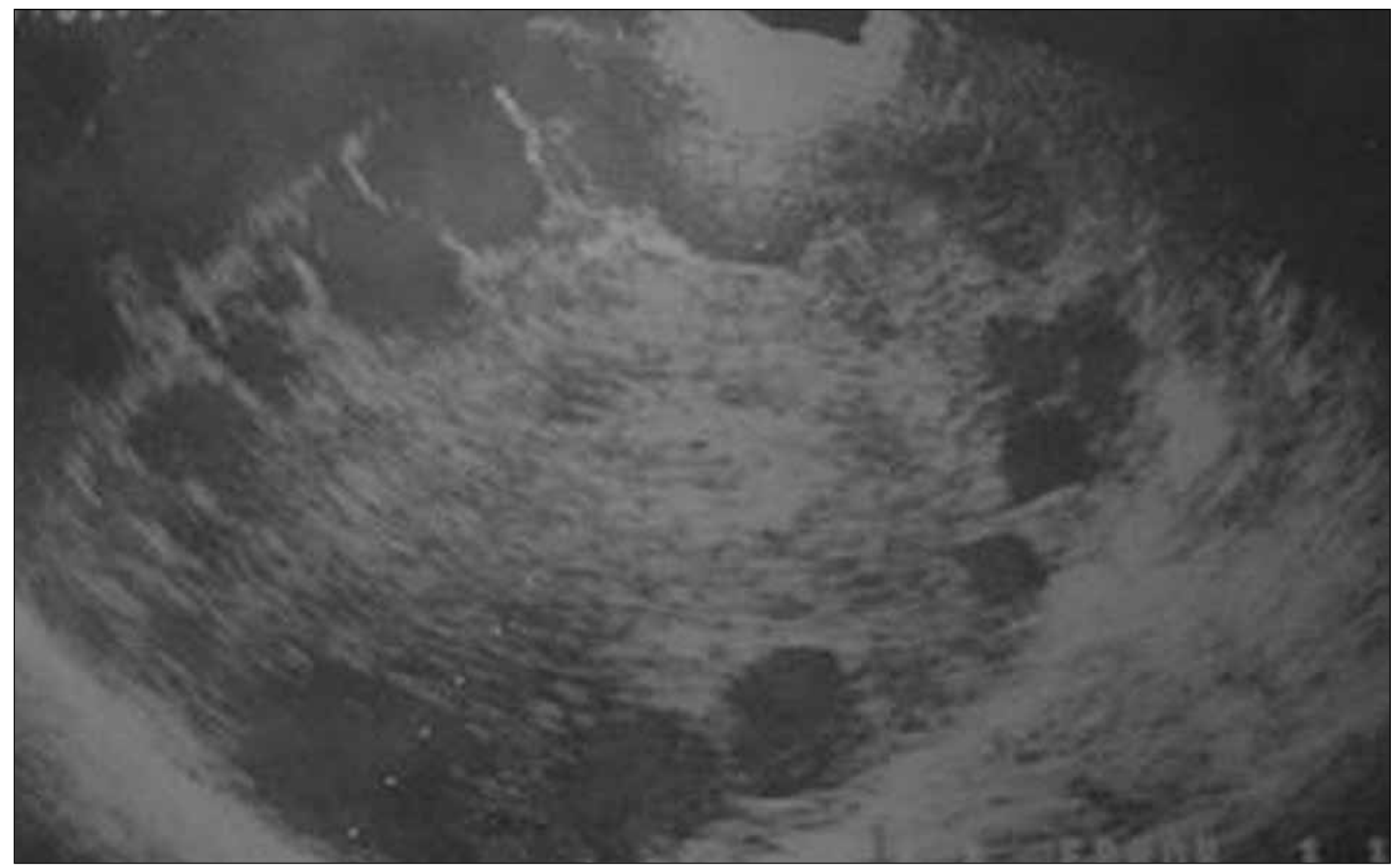

Fig. 2. Aspect échographique d'un kyste hydatique hétérogène avec quelques vésicules filles de type 4.

notre série, l'âge moyen de découverte était de 56,13 ans. Ce fait pourrait s'expliquer par la pauvreté de la population, I'inaccessibilité géographique des installations sanitaires dans la région de Marrakech et le retard de consultation.

Le KHR est habituellement unique et unilatéral, avec une prédominance du rein gauche. Aucun de nos malades ne présentait de localisation rénale bilatérale du kyste ni d'autre localisation viscérale associée.

Du fait de son développement rétropéritonéal, le KHR se caractérise par sa latence clinique, ce qui explique sa découverte fortuite chez deux de nos patients. Le syndrome douloureux a cependant été le principal symptôme révélateur dans $60 \%$ des cas de notre série, ce qui corrobore les résultats d'autres études, dans lesquelles la douleur s'est révélée le principal symptôme dans de $62,8 \%$ à $90 \%$ des cas $^{5,7}$. Le syndrome tumoral a été constaté respectivement dans $13,33 \%, 25,7 \%$ et $84 \%$ des cas dans les séries de Beddouch ${ }^{3}$, de Fekek ${ }^{4}$ et d'Ameur ${ }^{7}$. Ce fut également le cas dans notre série où 2 patients $(13,33 \%)$ ont présenté une masse lombaire à l'examen clinique. Les autres signes, tels que la fièvre, l'hypertension artérielle et les symptômes urinaires, restent rares. L'hydaturie, seul signe spécifique traduisant l'ouverture du kyste hydatique dans les voies excrétrices, n'a été signalée que par un seul de nos malades.

Le bilan radiologique reste l'élément essentiel du diagnostic. Selon HETET et coll., I'arbre urinaire sans préparation permet de déceler des calcifications de l'aire rénale dans de $15 \%$ à $60 \%$ des cas $^{8}$. Ces calcifications sont évocatrices, mais non spécifiques, car on a noté dans $2 \%$ des cas des kystes séreux et dans de $10 \%$ à $15 \%$ des cas, des tumeurs malignes. Selon certains auteurs, les calcifications ne traduisent nullement la mort du kyste ${ }^{8}$. Deux des 8 arbres urinaires sans préparation demandés pour nos patients ont révélé des calcifications, soit 25 \%. L'échographie possède un taux de

\section{Tableau 1. Les kystes selon la classification de Gharbi}

\begin{tabular}{ll}
\hline Type 1 & $\begin{array}{l}\text { Collection liquidienne pure nettement délimitée chez } \\
1 \text { patient }\end{array}$ \\
Type 2 & $\begin{array}{l}\text { Collection liquidienne à paroi dédoublée chez } 4 \\
\text { patients }\end{array}$ \\
Type 3 & $\begin{array}{l}\text { Collection liquidienne cloisonnée multivésiculaire } \\
\text { chez } 4 \text { patients }\end{array}$ \\
Type 4 & Contenu hétérogène chez 4 patients \\
Type 5 & KHR totalement calcifié chez 2 patients \\
\hline
\end{tabular}


El Harrech et al.

\begin{tabular}{lccccc}
\hline \multicolumn{6}{l}{ Tableau 2. Caractéristiques des patients, des kystes et des traitements } \\
\hline Patient & Âge (en années) & Sexe & Type de kyste & Taille du kyste & Traitement \\
\hline $\mathbf{1}$ & 54 & Homme & Type 4 & $100 \mathrm{~mm}$ & Résection du dôme saillant et traitement médical \\
$\mathbf{2}$ & 43 & Homme & Type 2 & $40 \mathrm{~mm}$ & Résection du dôme saillant \\
$\mathbf{3}$ & 33 & Homme & Type 4 & $50 \mathrm{~mm}$ & Résection du dôme saillant \\
$\mathbf{4}$ & 72 & Femme & Type 1 & $40 \mathrm{~mm}$ & Résection du dôme saillant \\
$\mathbf{5}$ & 62 & Homme & Type 2 & $75 \mathrm{~mm}$ & Résection du dôme saillant \\
$\mathbf{6}$ & 57 & Femme & Type 3 & $120 \mathrm{~mm}$ & Néphrectomie totale \\
$\mathbf{7}$ & 54 & Femme & Type 3 & $90 \mathrm{~mm}$ & Résection du dôme saillant \\
$\mathbf{8}$ & 38 & Homme & Type 5 & $55 \mathrm{~mm}$ & Abstention thérapeutique \\
$\mathbf{9}$ & 70 & Homme & Type 2 & $58 \mathrm{~mm}$ & Résection du dôme saillant \\
$\mathbf{1 0}$ & 64 & Homme & Type 3 & $60 \mathrm{~mm}$ & Résection du dôme saillant \\
$\mathbf{1 1}$ & 48 & Homme & Type 4 & $40 \mathrm{~mm}$ & Résection du dôme saillant \\
$\mathbf{1 2}$ & 40 & Homme & Type 5 & $170 \mathrm{~mm}$ & Abstention thérapeutique \\
$\mathbf{1 3}$ & 61 & Homme & Type 2 & $90 \mathrm{~mm}$ & Résection du dôme saillant et traitement médical \\
$\mathbf{1 4}$ & 53 & Homme & Type 4 & $100 \mathrm{~mm}$ & Résection du dôme saillant et traitement médical \\
$\mathbf{1 5}$ & 60 & Homme & Type 3 & $120 \mathrm{~mm}$ & Néphrectomie partielle \\
\hline
\end{tabular}

fiabilité de l'ordre de $80 \%$, même en cas de rupture du kyste dans les voies urinaires ${ }^{9-12}$. Les différents aspects échographiques ont été regroupés dans la classification de Gharbi. Cette classification, bien que largement utilisée, tend à être remplacée peu à peu par la classification internationale du non kyste hydatique de manière générale, ou classification de l'Organisation mondiale de la Santé ${ }^{13}$.

Dans notre série, les kystes de type 2, 3 et 4 se sont révélés les plus fréquents. La TDM est l'examen de deuxième intention et complète, dans la majorité des cas, l'échographie sans réellement décrire d'aspects caractéristiques. Elle montre une masse de densité liquidienne avec un halo périphérique hypodense. Elle définit au mieux le siège du kyste et son extension, de même que ses rapports avec la voie excrétrice. Pourtant, certains auteurs jugent que la TDM n'est indispensable qu'en cas de kyste hydatique de type 4 ou 5, dont le contenu ne peut être correctement analysé au moyen de l'échographie seule $e^{8,9}$.

L'hyperéosinophilie n'est pas un symptôme spécifique ni constant, et n'est présente que dans de $20 \%$ à $50 \%$ des cas, selon les séries ${ }^{14,15}$. Elle a été observée chez $20 \%$ de nos patients. L'hémagglutination indirecte est la réaction la plus sensible ${ }^{14,16}$, à raison $d^{\prime}$ un taux de sensibilité de $70 \%$.

En ce qui concerne l'aspect thérapeutique, il n'existe pas de traitement «parfait », et aucun essai clinique n'a comparé les différents modes thérapeutiques. En raison de la rareté de la localisation rénale, les recommandations générales sont moins pertinentes pour le KHR que pour le kyste hydatique hépatique.

La chirurgie est souvent considérée comme le traitement de choix des KHR. Pour de nombreuses équipes, la résection du dôme saillant est la méthode chirurgicale à privilégier, car elle est simple et rapide à réaliser, sans compter qu'elle entraîne moins de complications postopératoires ${ }^{7,16-18}$. Elle consiste à enlever la partie extériorisée, superficielle et avasculaire du périkyste. On se contente de pratiquer ce geste lorsque les parois de la cavité résiduelle sont souples.

La résection du dôme saillant donne d'excellents résultats et permet une bonne réexpansion du parenchyme réna $\left.\right|^{8,16-18}$. Dans notre série, 11 patients $(73,3 \%)$ ont subi une résection du dôme saillant sans aucun incident peropératoire - ni hémorragie ni plaie digestive ou vasculaire. Aucune récidive n'a été signalée après la chirurgie.

La périkystectomie totale est souvent difficile à réaliser, vu le risque hémorragique et le risque de communication avec la voie excrétrice. Aucun de nos malades n'a subi cette intervention. La néphrectomie partielle est recommandée par quelques auteurs en cas de kyste volumineux. Cependant, pour la plupart des équipes, elle paraît injustifiée. Elle a été réalisée chez un seul patient de notre étude. La néphrectomie totale n'est pratiquée, quant à elle, qu'en cas de rein détruit par un KHR volumineux ou de suppuration majeure.

La chirurgie laparoscopique a été utilisée avec succès ces dernières années pour le traitement des KHR. Les deux voies, transpéritonéale et rétropéritonéale, ont été essayées. Cependant, la voie transpéritonéale présente un risque d'essaimage de la grande cavité péritonéale ${ }^{19}$, et des études supplémentaires sont nécessaires pour l'évaluation de cette voie d'abord.

Malgré son efficacité éprouvée, le traitement percutané du kyste hydatique ne suscite pas I'unanimité. L'une des principales raisons de cette réticence est le risque de réaction anaphylactique qu'il sous-tend. Cependant, dans une vaste revue récente ${ }^{20}$, le taux de mortalité global d'origine 
anaphylactique s'est établi à 0,03\% (soit 2 malades sur 5 943), et le taux de réactions réversibles s'est chiffré à uniquement $1,7 \%$. Notons que ce risque de mortalité est presque identique à celui d'une prise médicamenteuse, qui est de l'ordre de $0,01 \%{ }^{21}$. Un patient non inclus dans cette étude a subi un traitement par néphrolithotomie percutanée modifiée sans complications peropératoires ni récidive sur une période d'un $\mathrm{an}^{22}$.

Pour la plupart des auteurs, le traitement médical seul est insuffisant, et son efficacité reste controversée. Il est indiqué chez les patients inopérables ou ayant des kystes multiples ${ }^{23}$.

L'abstention thérapeutique a été l'attitude adoptée par 2 de nos patients porteurs d'un KHR de type 5. Dans ce genre de situation, une surveillance régulière est obligatoire. L'abstention thérapeutique pourrait être indiquée chez un sujet âgé, porteur d'un kyste asymptomatique de petite taille et non évolutif, présentant un faible taux d'immunoglobulines spécifiques.

\section{Conclusion}

Le KHR est rare et sa sémiologie est variable. Le diagnostic est essentiellement radiologique. L'apport de la biologie est modeste en raison de sa non-spécificité. Si la chirurgie est actuellement le traitement de choix, de nouvelles perspectives mini-invasives restent à explorer, tout particulièrement les traitements laparoscopique et percutané.

Competing interests: Dr. El Harrech, Dr. Abakka, Dr. El Anzaoui, Dr. Ghoundale and Dr. Touiti all declare no competing financial or personal interests.

This paper has been peer-reviewed.

\section{Références}

1. Vuitton DA. The WHO Informal Working Group on Echinococcosis. Coordinating Board of the WHO-IWGE. Parassitologia 1997;39:349-53.
2. Gogus $C$, Safak $M$, Baltaci $S$, et al. Isolated renal hydatidosis: Experience with 20 cases. J Urol 2003;169:186-9. http://dx.doi.org/10.1016/S0022-5347(05)64064-5

3. Beddouch A, Ait Houssa M, Alkandry S, et al. Renal hydatid cyst. Apropos of 22 cases. J Urol (Paris) 1994; 100:304-6.

4. Fekak H, Bennani S, Rabii R, et al. Hydatic kidney cyst: 90 case reports. Ann Urol (Paris) 2003;37:85-9. http://dx.doi.org/10.1016/S0003-4401 (03)00026-3

5. Bellil S, Limaiem F, Bellil K, et al. Épidémiologie des kystes hydatiques extrapulmonaires : 265 cas en Tunisie. Médecine et maladies infectieuses 2009;39:341-3.

6. Amrani A, Zerhouni $\mathrm{H}$, Benabdallah $\mathrm{FF}$, et al. Renal hydatid cyst in children: Report of 6 cases. Ann Urol (Paris) 2003;37:8-12. http://dx.doi.org/10.1016/S0003-4401 (02)00009-8

7. Ameur $A$, Lezrek $M$, Boumdin $H$, et al. Hydatid cyst of the kidney based on a series of 34 cases. Prog Urol 2002;12:409-14.

8. Hétet JF, Vincendeau S, Rigaud J, et al. Renal hydatid cyst: Preoperative diagnosis and therapeutic implications [in French]. Prog Urol 2004;14:427-32.

9. Hasni Bouraoui I, Jemni H, Arifa N, et al. Imaging of renal hydatid cyst based on a series of 41 cases [in French]. Prog Urol 2006;16:139-44.

10. Abi F, el Fares F, Khaiz D, et al. Unusual localizations of hydatid cysts. Apropos of 40 cases. J Chir (Paris) 1989;126:307-12.

11. Afsar $\mathrm{H}$, Yagci F, Meto $\mathrm{S}$, et al. Hydatid disease of the kidney: Evaluation and features of diagnostic procedures. J Urol 1994;151:567-70.

12. Kilinc, $M, O$ dev $K$, Güngör $S$, et al. Sonographic diagnosis of the ruptured hydatid cyst of the kidney. Eur Radiol 1997;7:392-4. http://dx.doi.org/10.1007/s003300050172

13. WHO Informal Working Group. International classification of ultrasound images in cystic echinococcosis for application in clinical and field epidemiological settings. Acta Trop 2003;85:253-61. http://dx.doi. org/10.1016/50001-706X(02)00223-1

14. Göğüş C, Safak M, Baltaci S, et al. Isolated renal hydatidosis: Experience with 20 cases. J Urol 2003;169:186-9. http://dx.doi.org/10.1016/S0022-5347(05)64064-5

15. Idrissi S. Kyste hydatique du rein : à propos de 69 cas [dissertation]. Casablanca, Morocco; 1998.

16. Horchani A, Nouira Y, Kbaier I, et al. Hydatid cyst of the kidney. A report of 147 controlled cases. Eur Urol 2000;38:461-7. http://dx.doi.org/10.1159/000020325

17. Benchekroun A, Lachkar A, Soumana A, et al. Hydatid cyst of the kidney. Report of 45 cases. Ann Urol (Paris) 1999;33:19-24.

18. Imerli S, Ayed M, Horchani A, et al. Hydatid cyst of the kidney: Diagnosis and treatment. World I Surg 2001;25:68-74. htrp://dx.doi.org/10.1007/s002680020009

19. Khan $M$, Saijad Nazir $S$, Ahangar $S$, et al. Retroperitoneal laparoscopy for the management of renal hydatid cyst. Int I Surg 2010;8:266-8. http://dx.doi.org/10.1016/i.ijsu.2010.02.010

20. Neumayr A, Troia $G$, de Bernardis C, et al. Justified concern or exaggerated fear: The risk of anaphylaxis in percutaneous treatment of cystic echinococcosis-a systematic literature review. PLOS Negl Trop Dis 2011:5:e1154. http://dx.doi.org/10.1371/journal.pntd.0001154

21. Vervloet D, Durham S. Adverse reactions to drugs. BMJ 1998;316:1511-4

22. El Harrech Y, Abbaka N, Ghoundale 0, et al. PCNL approach for treatment of hydatid cysts of the kidney: A new percutaneous treatment. Urol J 2012;9:606-10.

23. Arif SH, Shams-Ul-Bari, Wani NA, et al. Albendazole as an adjuvant to the standard surgical management of hydatid cyst liver. Int I Surg 2008;6:448-51. http://dx.doi.org/10.1016/i.ijsu.2008.08.003

Correspondence: Dr. Youness El Harrech, Service d'urologie, Hôpital militaire Avicenne, Marrakech, Maroc; youness.elharrech@gmail.com 